\title{
Equidistribution for meromorphic maps with dominant topological degree
}

\author{
Tien-Cuong Dinh, Viêt-Anh Nguyên and Tuyen Trung Truong
}

July 25, 2018

\begin{abstract}
Let $f$ be a meromorphic self-map on a compact Kähler manifold whose topological degree is strictly larger than the other dynamical degrees. We show that repelling periodic points are equidistributed with respect to the equilibrium measure of $f$. We also describe the exceptional set of points whose backward orbits are not equidistributed.
\end{abstract}

Classification AMS 2010: 37F10, 32U40, 32H50

Keywords: meromorphic self-map, periodic point, equidistribution, exceptional set, tangent current.

\section{Introduction}

Let $X$ be a compact Kähler manifold of dimension $k$ and $\omega$ a Kähler form on $X$ so normalized that $\omega^{k}$ defines a probability measure on $X$. Let $f: X \rightarrow X$ be a meromorphic map. We always assume that $f$ is dominant, i.e. its image contains a non-empty open subset of $X$. The iterate of order $n$ of $f$ is defined by $f^{n}:=f \circ \cdots \circ f, n$ times, on a dense Zariski open set and extends to a dominant meromorphic map on $X$.

Let $d_{p}(f)$ (or $d_{p}$ if there is no possible confusion), $0 \leq p \leq k$, the dynamical degree of order $p$ of $f$. This is a bi-meromorphic invariant which measures the norm growth of the operators $\left(f^{n}\right)^{*}$ acting on the Hodge cohomology group $H^{p, p}(X, \mathbb{C})$ when $n$ tends to infinity, see Section 2 for details. We always have $d_{0}(f)=1$. The last dynamical degree $d_{k}(f)$ is the topological degree of $f$ : it is equal to the number of points in a generic fiber of $f$. We also denote it by $d_{t}(f)$ or simply by $d_{t}$. Throughout the paper, we assume that $f$ is with dominant topological degree 1 in the sense that $d_{t}>d_{p}$ for every $0 \leq p \leq k-1$.

\footnotetext{
${ }^{1}$ In some references, such a map is said to be with large topological degree; we think the word "dominant" is more appropriate.
} 
It is well-known that for such a map $f$, the following weak limit of probability measures

$$
\mu:=\lim _{n \rightarrow \infty} \frac{1}{d_{t}^{n}}\left(f^{n}\right)^{*} \omega^{k}
$$

exists. The probability measure $\mu$ is called the equilibrium measure of $f$. It has no mass on proper analytic subsets of $X$, is totally invariant: $d_{t}^{-1} f^{*}(\mu)=f_{*}(\mu)=\mu$ and is exponentially mixing. The measure $\mu$ is also the unique invariant measure with maximal entropy $\log d_{t}$. We refer the reader to [12, 13, 19] for details.

The first main result of this article is the following result.

Theorem 1.1. Let $f: X \rightarrow X$ be a meromorphic map with dominant topological degree $d_{t}$. Let $\mu$ be the equilibrium measure of $f$. Let $P_{n}$ (resp. $R P_{n}$ ) denote the set of isolated periodic (resp. repelling periodic) points of period $n$. Let $Q_{n}$ denote either $P_{n}, R P_{n}$ or their intersections with the support of $\mu$. Then $Q_{n}$ is asymptotically equidistributed with respect to $\mu$ : we have

$$
\frac{1}{d_{t}^{n}} \sum_{a \in Q_{n}} \delta_{a} \rightarrow \mu \quad \text { as } n \rightarrow \infty,
$$

where $\delta_{a}$ denotes the Dirac mass at a. In particular, we have $\# Q_{n}=d_{t}^{n}+o\left(d_{t}^{n}\right)$ as $n \rightarrow \infty$.

The last assertion in the above theorem is an important point in our proof. Indeed, when $f$ admits positive dimensional analytic sets of periodic points, the classical Lefschetz formula does not allow to estimate the number of isolated periodic points. The upper bound $\# Q_{n} \leq d_{t}^{n}+o\left(d_{t}^{n}\right)$ is, in fact, obtained using a very recent theory of density of positive closed currents developed by Sibony and the first author in [15].

For the rest of the proof, we need to construct enough repelling periodic points on the support of $\mu$. For this purpose, we will construct in Section 3 enough good inverse branches of balls for $f^{n}$ with controlled size, see Proposition 3.1 below. The construction of inverse branches for holomorphic discs in projective manifolds can be obtained using a method developed by Briend-Duval in [3. Here we follow the approach developed by Dinh-Sibony in [10] which also allows to carry out such a construction for discs as well as balls in Kähler manifolds. Then an idea of Buff allows to obtain repelling periodic points [5. The presence of indeterminacy sets for meromorphic maps is the source of several delicate technical points in the proof. For example, the obstruction to the existence of inverse branches for balls, at least in our approach, may be larger than the orbits of critical values and of indeterminacy loci. We will construct and use a positive closed $(1,1)$-current $R$ which allows to control this obstruction.

Note that when $X$ is a projective manifold, a weaker version of Theorem 1.1 was stated in [19]. The author's proof is, however, based on Lemma 3.3 therein 
whose proof is incomplete (the radius $r_{\epsilon}$ therein depends on the integer $l$ ) and the statement is still an open question. When $f$ is a holomorphic endomorphism of $\mathbb{P}^{k}$, the above theorem was obtained by Briend-Duval in [2]. Their proof uses strongly the Hölder continuity of the dynamical Green function. For meromorphic maps, the dynamical Green function, even when it exists, is in general not continuous. The same result for polynomial-like maps of dominant topological degree, in particular for a large family of rational maps on $\mathbb{P}^{k}$, was obtained by Sibony and the first author [10]. For the case of dimension 1, see Brolin, Freire-Lopes-Mañé, Lyubich and Tortrat [4, 17, 23, 29].

Our construction of inverse branches of balls also allows to study the equidistribution of preimages of points by $f^{n}$. Let $I^{\prime}$ denote the second indeterminacy set of $f$, i.e. the set of points $z$ such that $f^{-1}(z)$ has positive dimension. It is an analytic set of codimension at least equal to 2 . The Zariski open set $X \backslash I^{\prime}$ is the set of points $a$ such that the fiber $f^{-1}(a)$ contains exactly $d_{t}$ points counted with multiplicity, see Section 2 for the definition of the action of $f$ and $f^{-1}$ on subsets of $X$.

Define $I_{0}^{\prime}:=I^{\prime}, I_{n+1}^{\prime}:=I_{0}^{\prime} \cup f\left(I_{n}^{\prime}\right)$ for $n \geq 0$ and $I_{\infty}^{\prime}:=\cup_{n \geq 0} I_{n}^{\prime}$. Note that the set $I_{\infty}^{\prime}$ is characterized by the following property: the sequence of probability measures

$$
\mu_{0}^{a}:=\delta_{a}, \quad \mu_{n+1}^{a}:=d_{t}^{-1} f^{*}\left(\mu_{n}^{a}\right) \quad \text { for } n \geq 0
$$

is well-defined if and only if $a \notin I_{\infty}^{\prime}$. We have $\mu_{n}^{a}=d_{t}^{-n}\left(f^{n}\right)^{*}\left(\delta_{a}\right)$. So $\mu_{n}^{a}$ is the probability measure equidistributed on the fiber $f^{-n}(a)$ where the points in this fiber are counted with multiplicity. One has to distinguish $I_{\infty}^{\prime}$ with the set $\cup_{n \geq 0} f^{n}\left(I^{\prime}\right)$ which is a priori smaller.

Let $I$ be the (first) indeterminacy set of $f$. Define also $I_{0}:=I, I_{n+1}:=$ $I_{0} \cup f\left(I_{n}\right)$ for $n \geq 0$ and $I_{\infty}:=\cup_{n \geq 0} I_{n}$. The set $I_{\infty} \backslash I_{\infty}^{\prime}$ consists of points $a \notin I_{\infty}^{\prime}$ such that the support of $\mu_{n}^{a}$ intersects $I$ for some $n \geq 0$. We will consider $a \notin I_{\infty} \cup I_{\infty}^{\prime}$. Here is the second main result in this paper.

Theorem 1.2. Let $f: X \rightarrow X$ and $\mu$ be as in the statement of Theorem 1.1. Then there is a (possibly empty) proper analytic set $\mathscr{E}$ of $X$ such that for a $\notin$ $I_{\infty} \cup I_{\infty}^{\prime}$ we have

$$
\frac{1}{d_{t}^{n}}\left(f^{n}\right)^{*} \delta_{a} \rightarrow \mu \quad \text { as } \quad n \rightarrow \infty
$$

if and only if $a \notin \mathscr{E}$.

When $X$ is projective, it was shown by Guedj in [19] that $\mathscr{E}$ is a finite or countable union of analytic sets, see also [13] for the case of compact Kähler manifolds. The above theorem was obtained for holomorphic endomorphisms of $\mathbb{P}^{k}$ in [3, 10, 16]. It also holds for polynomial-like maps with dominant topological degree. For the case of dimension 1, see [4, 17, 23, 29]. Note that there are many meromorphic maps with $I^{\prime}=\varnothing$ which are not holomorphic. For example, if $g: \widehat{\mathbb{P}}^{k} \rightarrow \mathbb{P}^{k}$ is a blow-up of $\mathbb{P}^{k}$ and $\pi: \widehat{\mathbb{P}}^{k} \rightarrow \mathbb{P}^{k}$ is a finite holomorphic map, 
then $\pi \circ g^{-1}$ is not holomorphic but its second indeterminacy set is empty. For holomorphic maps on $\mathbb{P}^{k}$, we have $I=I^{\prime}=\varnothing$.

The article is organized as follows. In Section 2 we prepare the background and fix some terminology as well as recall auxiliary results concerning the actions of meromorphic maps on currents and on cohomology and the theory of density for positive closed currents. Section 3 is devoted to the construction of good inverse branches of iterates of $f$, which is one of the main tools of our work. Using these inverse branches we prove Theorems 1.2 in Section 4. After establishing an upper bound on the number of isolated periodic points, we prove Theorem 1.1 in Section 5. In the same section, we also explain how to obtain a lower bound for Lyapounov exponents of $\mu$ from our construction of inverse branches for balls.

Acknowledgement. The paper was partially prepared during the visit of the second author at the University of Cologne upon a Humboldt foundation research grant, and during the visit of the third author at the University of Paris 6 and at the University of Paris 11 (Orsay). They would like to express their gratitude to these organizations for hospitality and for financial support. The second author also would like to thank Professor George Marinescu for kind help.

\section{Meromorphic maps and currents}

In this section we define various operations for meromorphic maps and positive closed currents on compact Kähler manifolds. We also recall some elements of the theory of density of positive closed currents and establish a preparatory result. We refer the reader to Demailly [7], Dinh-Sibony [14, 24] and Voisin [30] for basic notions on positive closed currents and quasi-plurisubharmonic (quasi-p.s.h. for short) functions and basic facts on Kähler geometry.

Let $X$ be a compact Kähler manifold of dimension $k$ and $\omega$ a Kähler form on $X$ as above. If $T$ is a current on $X$ and $\varphi$ is a test form of right degree, the pairing $\langle T, \varphi\rangle$ denotes the value of $T$ at $\varphi$. If $T$ is a positive $(p, p)$-current on $X$, its mass is given by the formula

$$
\|T\|:=\left\langle T, \omega^{k-p}\right\rangle
$$

Note that when $T$ is, moreover, closed, its mass depends only on its cohomology class $\{T\}$ in $H^{p, p}(X, \mathbb{R})$. Here $H^{p, q}(X, \mathbb{C})$ denotes the Hodge cohomology group of bidegree $(p, q)$ of $X$ and $H^{p, p}(X, \mathbb{R}):=H^{p, p}(X, \mathbb{C}) \cap H^{2 p}(X, \mathbb{R})$.

We will write $T \leq T^{\prime}$ and $T^{\prime} \geq T$ for two real $(p, p)$-currents $T, T^{\prime}$ if $T^{\prime}-T$ is a positive current. We also write $c \leq c^{\prime}$ and $c^{\prime} \geq c$ for $c, c^{\prime} \in H^{p, p}(X, \mathbb{R})$ when $c^{\prime}-c$ is the class of a positive closed $(p, p)$-current. If $V$ is an analytic subset of pure dimension $k-p$ in $X$, denote by $[V]$ the positive closed current of integration on $V$ and $\{V\}$ its cohomology class in $H^{p, p}(X, \mathbb{R})$. The cup-product in $H^{*}(X, \mathbb{C})$ is denoted by $\smile$. 
Consider now a dominant meromorphic map $f: X \rightarrow X$. Recall that $f$ is holomorphic on a Zariski open set and the closure $\Gamma$ of its graph in $X \times X$ is an irreducible analytic subset of dimension $k$. Let $\pi_{1}$ and $\pi_{2}$ denote the canonical projections from $X \times X$ onto its factors. If $A$ is a subset of $X$ define $f(A):=$ $\pi_{2}\left(\pi_{1}^{-1}(A) \cap \Gamma\right)$ and $f^{-1}(A):=\pi_{1}\left(\pi_{2}^{-1}(A) \cap \Gamma\right)$. The (first) indeterminacy set $I$ of $f$ is the complement of the set of all points $z \in X$ such that $f(z)$ is of dimension 0 , or equivalently, that $f(z)$ contains only one point. The second indeterminacy set $I^{\prime}$ of $f$ is the complement of the set of all points $z$ such that $f^{-1}(z)$ is of dimension 0 , or equivalently, that $f^{-1}(z)$ contains exactly $d_{t}$ points counted with multiplicity. Both $I$ and $I^{\prime}$ are analytic subsets of $X$ of codimension at least equal to 2.

The map $f$ induces linear operators on forms and currents. The presence of indeterminacy locus makes these operators more delicate to handle. If $\varphi$ is a smooth $(p, q)$-form on $X$, then $f^{*}(\varphi)$ is the $(p, q)$-current defined by

$$
f^{*}(\varphi):=\left(\pi_{1}\right)_{*}\left(\pi_{2}^{*}(\varphi) \wedge[\Gamma]\right) .
$$

It is not difficult to see that $f^{*}(\varphi)$ is an $L^{1}$-form smooth outside $I$. Its singularities along $I$ do not allow to iterate the operation in the same way. Nevertheless, the operation commutes with $\partial$ and $\bar{\partial}$. In particular, when $\varphi$ is closed or exact, so is $f^{*}(\varphi)$. Therefore, $f^{*}$ induces a linear operator on $H^{p, q}(X, \mathbb{C})$. We can iterate the later operator but in general we do not have $\left(f^{*}\right)^{n}=\left(f^{n}\right)^{*}$.

In the same way, the $(p, q)$-current $f_{*}(\varphi)$ is defined by

$$
f_{*}(\varphi):=\left(\pi_{2}\right)_{*}\left(\pi_{1}^{*}(\varphi) \wedge[\Gamma]\right) .
$$

This is an $L^{1}$-form smooth outside the critical values of $\pi_{2 \mid \Gamma}$. The operator $f_{*}$ also commutes with $\partial, \bar{\partial}$ and induces a linear operator $f_{*}$ on $H^{p, q}(X, \mathbb{C})$.

Recall that the dynamical degree of order $p$ of $f$ is defined by

$$
\begin{aligned}
d_{p} & =\lim _{n \rightarrow \infty}\left\|\left(f^{n}\right)^{*}\left(\omega^{p}\right)\right\|^{1 / n}=\lim _{n \rightarrow \infty}\left\|\left(f^{n}\right)_{*}\left(\omega^{k-p}\right)\right\|^{1 / n} \\
& =\lim _{n \rightarrow \infty}\left\|\left(f^{n}\right)^{*}\right\|_{H^{p, p}(X, \mathbb{C})}^{1 / n}=\lim _{n \rightarrow \infty}\left\|\left(f^{n}\right)_{*}\right\|_{H^{k-p, k-p}(X, \mathbb{C})}^{1 / n} .
\end{aligned}
$$

The above limits exist and do not depend on the choice of $\omega$ nor on the norm fixed for $H^{*}(X, \mathbb{C})$. They are bi-meromorphic invariants and play a central role in complex dynamics, see [12] for details. Recall also that a mixed version of the Hodge-Riemann theorem [9, 18, 22, 27, 28] implies that $p \mapsto \log d_{p}$ is concave, i.e. $d_{p}^{2} \geq d_{p-1} d_{p+1}$. So $f$ has dominant topological degree if and only if $d_{t}>d_{k-1}$.

We will now consider two particular cases of the pull-back operator $f^{*}$ on currents that will be used later on. If $\phi$ is a continuous function on $X$ then $f_{*}(\phi)$ is a bounded function on $X$ which is continuous outside $I^{\prime}$. Therefore, if $\nu$ is a positive measure without mass on $I^{\prime}$ we can define

$$
\left\langle f^{*}(\nu), \phi\right\rangle:=\left\langle\nu, f_{*}(\phi)\right\rangle \text {. }
$$


It is not difficult to see that $f^{*}(\nu)$ is a positive measure whose mass is equal to $d_{t}$ times the mass of $\nu$ since $\pi_{2}$ restricted to $\Gamma$ defines a ramified covering of degree $d_{t}$ over $X \backslash I^{\prime}$. If $\nu$ is the Dirac mass at a point $a \notin I^{\prime}$, then $f^{*}(\nu)$ is the sum of the Dirac masses on the fiber $f^{-1}(a)$ counted with multiplicity. If $\nu$ has no mass on $I$, the positive measure $f_{*}(\nu)$ given by

$$
\left\langle f_{*}(\nu), \phi\right\rangle:=\left\langle\nu, f^{*}(\phi)\right\rangle
$$

for every continuous function $\phi$ on $X$, is well-defined and has the same mass as $\nu$. If $\nu$ is the Dirac mass at $a \notin I$, then $f_{*}(\nu)$ is the Dirac mass at $f(a)$.

The second situation concerns positive closed $(1,1)$-currents. If $T$ is such a current on $X$, we can write $T=\alpha+d d^{c} u$ where $\alpha$ is a smooth closed $(1,1)$-form in the class $\{T\}$ and $u$ is a quasi-p.s.h. function. Then $u \circ \pi_{2}$ is a quasi-p.s.h. function on $\Gamma$ and we define

$$
f^{*}(T):=f^{*}(\alpha)+\left(\pi_{1}\right)_{*}\left(d d^{c}\left(u \circ \pi_{2 \mid \Gamma}\right)\right) .
$$

Using a local regularization of $T$, one can see that $f^{*}(T)$ is a positive closed $(1,1)$-current. The operator is linear and continuous on $T$. So using Demailly's regularization of $(1,1)$-currents on $X[6]$, we can easily check that the operator is compatible with cohomology, that is, we have $\left\{f^{*}(T)\right\}=f^{*}\{T\}$. The operator $f_{*}$ is defined in the same way on positive closed $(1,1)$-currents and is also compatible with the cohomology.

In the rest of this section, we recall basic facts on the theory of density of positive closed currents and give an abstract result which will allow us to bypass Lefschetz's fixed points formula in order to bound the number of periodic points. We will restrict ourselves to the simplest situation that is needed for the present work. We will come back to this subject in a forthcoming paper. The reader is invited to consult [15] for details.

Let $V$ be an irreducible submanifold of $X$ of dimension $l$. Let $\pi: E \rightarrow V$ denote the normal vector bundle of $V$ in $X$. For a point $a \in V$, if $\operatorname{Tan}_{a} X$ and $\operatorname{Tan}_{a} V$ denote respectively the tangent spaces of $X$ and of $V$ at $a$, the fiber $E_{a}:=$ $\pi^{-1}(a)$ of $E$ over $a$ is canonically identified with the quotient $\operatorname{space} \operatorname{Tan}_{a} X / \operatorname{Tan}_{a} V$. The zero section of $E$ is naturally identified with $V$. Denote by $\bar{E}$ the natural compactification of $E$, i.e. the projectivisation $\mathbb{P}(E \oplus \mathbb{C})$ of the vector bundle $E \oplus \mathbb{C}$, where $\mathbb{C}$ is the trivial line bundle over $V$. We still denote by $\pi$ the natural projection from $\bar{E}$ to $V$. Denote by $A_{\lambda}$ the multiplication by $\lambda$ on the fibers of $E$ where $\lambda \in \mathbb{C}^{*}$, i.e. $A_{\lambda}(u):=\lambda u, u \in E_{a}, a \in V$. This map extends to a holomorphic automorphism of $\bar{E}$.

Let $V_{0}$ be an open subset of $V$ which is naturally identified with an open subset of the section 0 in $E$. A diffeomorphism $\tau$ from a neighbourhood of $V_{0}$ in $X$ to a neighbourhood of $V_{0}$ in $E$ is called admissible if it satisfies essentially the following three conditions: the restriction of $\tau$ to $V_{0}$ is the identity, the differential of $\tau$ at each point $a \in V_{0}$ is $\mathbb{C}$-linear and the composition of

$$
E_{a} \hookrightarrow \operatorname{Tan}_{a}(E) \rightarrow \operatorname{Tan}_{a}(X) \rightarrow E_{a}
$$


is the identity, where the morphism $\operatorname{Tan}_{a}(E) \rightarrow \operatorname{Tan}_{a}(X)$ is given by the differential of $\tau^{-1}$ at $a$ and the other maps are the canonical ones, see [15] for details.

Note that an admissible map is not necessarily holomorphic. When $V_{0}$ is small enough, there are local holomorphic coordinates on a small neighbourhood $U$ of $V_{0}$ in $X$ so that over $V_{0}$ we identify naturally $E$ with $V_{0} \times \mathbb{C}^{k-l}$ and $U$ with an open neighbourhood of $V_{0} \times\{0\}$ in $V_{0} \times \mathbb{C}^{k-l}$ (we reduce $U$ if necessary). In this picture, the identity is a holomorphic admissible map. There always exist admissible maps for $V_{0}:=V$. However, such a global admissible map is rarely holomorphic.

Consider an admissible map $\tau$ as above. Let $T$ be a positive closed $(p, p)$ current on $X$ without mass on $V$ for simplicity. Define $T_{\lambda}:=\left(A_{\lambda}\right)_{*} \tau_{*}(T)$. The family $\left(T_{\lambda}\right)$ is relatively compact on $\pi^{-1}\left(V_{0}\right)$ when $\lambda \rightarrow \infty$ : we can extract convergent subsequences for $\lambda \rightarrow \infty$. The limit currents $R$ are positive closed $(p, p)$-currents without mass on $V$ which are $V$-conic, i.e. $\left(A_{\lambda}\right)_{*} R=R$ for any $\lambda \in \mathbb{C}^{*}$, in other words, $R$ is invariant by $A_{\lambda}$.

Such a current $R$ depends on the choice of $\lambda \rightarrow \infty$ but it is independent of the choice of $\tau$. This property gives us a large flexibility to work with admissible maps. In particular, using global admissible maps, we obtains positive closed $(p, p)$-currents $R$ on $\bar{E}$. It is also known that the cohomology class of $R$ depends on $T$ but does not depend on the choice of $R$. This class is denoted by $\kappa^{V}(T)$ and is called the total tangent class of $T$ with respect to $V$. The currents $R$ are called tangent currents of $T$ along $V$. The mass of $R$ and the norm of $\kappa^{V}(T)$ is bounded by a constant times the mass of $T$.

Let $-h$ denote the tautological $(1,1)$-class on $\bar{E}$. Recall that $H^{*}(\bar{E}, \mathbb{C})$ is a free $H^{*}(V, \mathbb{C})$-module generated by $1, h, \ldots, h^{k-l}$ (the fibers of $\bar{E}$ are of dimension $k-l)$. So we can write

$$
\kappa^{V}(T)=\sum_{j=\max (0, l-p)}^{\min (l, k-p)} \pi^{*}\left(\kappa_{j}^{V}(T)\right) \smile h^{j-l+p}
$$

where $\kappa_{j}^{V}(T)$ is a class in $H^{l-j, l-j}(V, \mathbb{C})$ with the convention that $\kappa_{j}^{V}(T)=0$ outside of the range $\max (0, l-p) \leq j \leq \min (l, k-p)$.

Let $s$ be the maximal integer such that $\kappa_{s}^{V}(T) \neq 0$. We call it the tangential $h$-dimension of $T$ along $V$. The class $\kappa_{s}^{V}(T)$ is pseudo-effective, i.e. contains a positive closed current on $V$. The tangential h-dimension of $T$ is also equal to the maximal integer $s \geq 0$ such that $R \wedge \pi^{*}\left(\omega_{V}^{s}\right) \neq 0$, where $\omega_{V}$ is any Kähler form on $V$. Moreover, if $T_{n}$ and $T$ are positive closed $(p, p)$-currents on $X$ such that $T_{n} \rightarrow T$, then $\kappa_{j}^{V}\left(T_{n}\right) \rightarrow 0$ for $j>s$ and any limit class of $\kappa_{s}^{V}\left(T_{n}\right)$ is pseudo-effective and is smaller than or equal to $\kappa_{s}^{V}(T)$.

The following result will allow us to bound the number of isolated periodic points of a meromorphic map. We identify here the cohomology group $H^{2 k}(X, \mathbb{C})$ with $\mathbb{C}$ using the integrals of top degree differential forms on $X$. 
Proposition 2.1. Let $\Gamma_{n}$ be complex subvarieties of pure dimension $k-l$ in $X$. Assume that there is a sequence of positive numbers $d_{n}$ such that $d_{n} \rightarrow \infty$ and $d_{n}^{-1}\left[\Gamma_{n}\right]$ converges to a positive closed $(l, l)$-current $T$ on $X$. Assume also that the h-tangent dimension of $T$ with respect to $V$ is 0 and that $\{T\} \smile\{V\}=1$. Then the number $\delta_{n}$ of isolated points in the intersection $\Gamma_{n} \cap V$, counted with multiplicity, satisfies $\delta_{n} \leq d_{n}+o\left(d_{n}\right)$ as $n \rightarrow \infty$.

We need the following lemma.

Lemma 2.2. Let $\Gamma$ be a subvariety of pure dimension $k-l$ in $X$. Let $a_{1}, \ldots, a_{N}$ be the isolated points in $\Gamma \cap V$ and $m_{i}$ the multiplicity of the intersection of $\Gamma \cap V$ at $a_{i}$. Then any tangent current of $[\Gamma]$ along $V$ is larger than or equal to $\sum m_{i}\left[\pi^{-1}\left(a_{i}\right)\right]$.

Proof. Consider a small open set $V_{0}$ in $V$ which contains only one point $a_{i}$. As above, we identify $E$ (resp. $\bar{E}$ ) over $V_{0}$ with $V_{0} \times \mathbb{C}^{k-l}\left(\right.$ resp. $\left.V_{0} \times \mathbb{P}^{k-l}\right)$, and a small neighbourhood of $V_{0}$ in $X$ with an open neighbourhood of $V_{0} \times\{0\}$ in $V_{0} \times \mathbb{C}^{k-l}$, and $\pi$ with the canonical projection of $V_{0} \times \mathbb{P}^{k-l}$ onto its first factor. The identity is then an admissible map. It is clear in this picture that any tangent current of $[\Gamma]$ along $V$ constructed as above is larger than or equal to $m_{i}\left[\pi^{*}\left(a_{i}\right)\right]$. The lemma follows.

Proof of Proposition 2.1. Define $T_{n}:=d_{n}^{-1}\left[\Gamma_{n}\right]$. Extracting a subsequence allows us to assume that $\kappa^{V}\left(T_{n}\right)$ converges to a class $\kappa$. Since the h-tangent dimension of $T$ is zero, the above discussion implies that $\kappa=\lambda c$, where $c$ is the class of a fiber of $\bar{E}$ and $\lambda$ is a positive number. We also have $\kappa^{V}(T)=\pi^{*}\left(\kappa_{0}^{V}(T)\right)$. In the above construction of $\kappa^{V}(T)$ with a global admissible map, we see that the de Rham cohomology class of $T_{\lambda}$ in a neighbourhood of $V_{0} \times\{0\}$ does not depend on $\lambda$ when $\lambda \rightarrow \infty$. It follows that $\{T\} \smile\{V\}=\kappa^{V}(T) \smile\{V\}$. This together with the hypothesis $\{T\} \smile\{V\}=1$ implies that $\kappa^{V}(T)=c$. The above discussion on the upper semi-continuity of $\kappa_{s}^{V}\left(T_{n}\right)$ implies that $\lambda \leq 1$.

By Lemma 2.2, we can write $\kappa^{V}\left(T_{n}\right)=\delta_{n} d_{n}^{-1} c+c_{n}$ where $c_{n}$ is a pseudoeffective class. Since $\kappa^{V}\left(T_{n}\right)$ converges to $\kappa=\lambda c$, we deduce that the cluster values of $c_{n}$ are also equal to positive constants times $c$ and then $\lim \sup \delta_{n} d_{n}^{-1} \leq$ $\lambda$. The proposition follows.

\section{Construction of good inverse branches}

Consider a map $f: X \rightarrow X$ as above with dominant topological degree. The purpose of this section is to construct for generic small balls an almost maximal number of inverse branches with respect to $f^{n}$ that we control the size.

Recall that $I, I^{\prime}, d_{p}, d_{t}, \Gamma$ denote the indeterminacy sets, the dynamical degree of order $p$, the topological degree and the closure of the graph of $f$ in $X \times X$. By definition, the dynamical degree of order $p$ and the topological degree of $f^{n}$ are 
equal to $d_{p}^{n}$ and $d_{t}^{n}$ respectively. Denote by $I\left(f^{n}\right), I^{\prime}\left(f^{n}\right), \Gamma_{n}$ the indeterminacy sets and the closure of the graph of $f^{n}$. The natural projections from $X \times X$ onto its factors are denoted by $\pi_{1}$ and $\pi_{2}$. Recall also that $I_{0}^{\prime}:=I^{\prime}, I_{n+1}^{\prime}:=I_{0}^{\prime} \cup f\left(I_{n}^{\prime}\right)$ for $n \geq 0$ and $I_{\infty}^{\prime}:=\cup_{n \geq 0} I_{n}^{\prime}$. One should distinguish $I_{\infty}^{\prime}$ with the set $\cup_{n \geq 0} f^{n}\left(I^{\prime}\right)$ and the union of $I^{\prime}\left(f^{n}\right)$ which are a priori smaller.

Choose an analytic subset $\Sigma_{0}$ of $X$ containing $I, I^{\prime}, f(I), f^{-1}\left(I^{\prime}\right)$ such that $\pi_{2}$ restricted to $\Gamma \backslash \pi_{2}^{-1}\left(\Sigma_{0}\right)$ defines an unramified covering over $X \backslash \Sigma_{0}$. Let $B$ be a connected subset of $X$, e.g. a holomorphic ball, a holomorphic disc or a family of discs through a point in $X$. We call ${ }^{2}$ an inverse branch of order $n$ of $B$ any continuous bijective map $g: B \rightarrow B_{-n}$ with $B_{-n} \subset X$ such that if we define $B_{-i}:=f\left(B_{-i-1}\right)$ with $0 \leq i \leq n-1$, then $B_{-i} \cap \Sigma_{0}=\varnothing$ for $0 \leq i \leq n$, $f: B_{-i} \rightarrow B_{-i+1}$ is a bijective map for $1 \leq i \leq n, B_{0}=B$ and $f^{n} \circ g=$ id on $B$.

Note that $f^{n-i} \circ g: B \rightarrow B_{-i}$ is an inverse branch of order $i$ of $B$ and $B$ admits at most $d_{t}^{n}$ inverse branches of order $n$. The condition $B_{-i} \cap \Sigma_{0}=\varnothing$ implies that the inverse branch can be extended to any small enough open set containing $B$ using local inverses of the map $f^{n}$. We say that the above inverse branch is of size smaller than $\lambda$ if the diameter of $B_{-n}$ is smaller than $\lambda$. We also call $B_{-n}$ the image of the inverse branch $g: B \rightarrow B_{-n}$.

Proposition 3.1. There is a positive closed $(1,1)$-current $R$ on $X$ satisfying the following property. Let $\epsilon, \nu$ be strictly positive numbers with $\nu \leq 1$ and let a be a point in $X$ such that the Lelong number $\nu(R, a)$ of $R$ at a is smaller than $\nu$. Then there is a constant $r>0$ such that the ball $B(a, r)$ of center $a$ and of radius $r$ admits at least $(1-\nu) d_{t}^{n}$ inverse branches of order $n$ and of size smaller than $\left(d_{k-1} / d_{t}+\epsilon\right)^{n / 2}$ for every $n \geq 0$.

A theorem by Siu says that $\{\nu(R, a) \geq c\}$ is a proper analytic subset of $X$ for every $c>0$ [26]. So the above proposition applies for generic points $a$ in $X$. We will see later in the construction of $R$ that the set $\{\nu(R, a)>0\}$ contains the orbits of the critical values and of the indeterminacy points which are obviously an obstruction to obtain inverse branches of balls. However, $\{\nu(R, a)>0\}$ contains a priori other analytic sets which are a less obvious obstruction to the existence of inverse branches. It can be seen as an accumulation locus of the orbits of the indeterminacy points. We give now the proof of the above proposition. The first step is to define the current $R$.

Recall that the operators $\left(f^{n}\right)_{*}$ act continuously on positive closed $(1,1)$ currents and these actions are compatible with the actions of $\left(f^{n}\right)_{*}$ on $H^{1,1}(X, \mathbb{R})$. If $T$ is a positive closed $(1,1)$-current on $X$, its mass depends only on the cohomology class $\{T\}$. Therefore, for a fixed norm on cohomology, the mass of $T$ is comparable with the norm of $\{T\}$. We then deduce the existence of a constant

\footnotetext{
${ }^{2}$ We can weaken the conditions in the definition but the ones given here are simple and sufficient for our purpose.
} 
$c_{0}>0$ independent of $T, f$ and $n$ such that

$$
\left\|\left(f^{n}\right)_{*}(T)\right\| \leq c_{0}\left\|\left(f^{n}\right)_{*}\right\|_{H^{1,1}(X, \mathbb{R})}\|T\| .
$$

By definition of $d_{k-1}$, we can fix an integer $N \geq 1$ large enough such that $c_{0}\left\|\left(f^{N}\right)_{*}\right\|_{H^{1,1}(X, \mathbb{R})}<\left(\theta d_{t}\right)^{N}$, where $d_{k-1} / d_{t}<\theta<d_{k-1} / d_{t}+\epsilon$ is any fixed constant strictly smaller than 1 .

Define $\Sigma_{i+1}:=f\left(\Sigma_{i}\right)$ for $0 \leq i \leq N-1$ and $\Sigma:=\cup_{0 \leq i \leq N} \Sigma_{i}$. So any connected and simply connected set outside $\Sigma$ admits the maximal number $d_{t}^{N}$ of inverse branches of order $N$ with images outside $\Sigma_{0}$. Choose a desingularization $\pi$ : $\widehat{\Gamma} \rightarrow \Gamma$ which is a composition of blow-ups of $\Gamma$ along smooth centers in or over $\pi_{1}^{-1}(\Sigma) \cap \Gamma$ and $\pi_{2}^{-1}(\Sigma) \cap \Gamma$. Define $\tau_{i}:=\pi_{i} \circ \pi$. We can choose $\pi$ so that $\tau_{1}^{-1}(\Sigma)$ and $\tau_{2}^{-1}(\Sigma)$ are of pure codimension 1 in $\widehat{\Gamma}$. By Blanchard's theorem [1], $\widehat{\Gamma}$ is a compact Kähler manifold. Fix a Kähler form $\widehat{\omega}$ on $\widehat{\Gamma}$ which is larger than $\tau_{i}^{*}(\omega)$. We also assume that $\widehat{\omega}$ is large enough so that each ball of radius 1 in $\widehat{\Gamma}$ with respect to the metric $\widehat{\omega}$ is contained in an open set biholomorphic to a ball in $\mathbb{C}^{k}$.

Denote by $\Sigma^{\prime}$ and $\Sigma^{\prime \prime}$ respectively the union of components of codimension 1 and the union of components of codimension $\geq 2$ of $\Sigma$. Define

$$
S_{0}:=c_{1} \theta^{-N} d_{t}^{N}\left(\left[\Sigma^{\prime}\right]+\left(\tau_{2}\right)_{*}(\widehat{\omega})\right), \quad S:=\sum_{n=0}^{N}\left(f_{*}\right)^{n}\left(S_{0}\right)
$$

and

$$
R:=8 \sum_{m \geq 0}\left(\theta d_{t}\right)^{-m N}\left(f^{N}\right)_{*}^{m}(S) .
$$

Here $c_{1} \geq \delta_{1}^{-1}$ is a constant satisfying Lemma 3.2 below, and $\delta_{1}$ is a constant whose exact value will be determined right after Lemma 3.4 below. By definition of $\theta$, the last current is well-defined. Note that one has to distinguish the operators $\left(f^{N}\right)_{*}^{m}$ and $\left(f^{N m}\right)_{*}$. The orbit of $\Sigma$ is the obstruction to construct inverse branches of balls. The following lemma shows that it is visible using the current $R$.

Lemma 3.2. If $c_{1}$ is large enough, then for every $a \in \Sigma$ the Lelong number $\nu\left(S_{0}, a\right)$ of $S_{0}$ at a is larger than 1 .

Proof. The lemma is clear for $a \in \Sigma^{\prime}$. Consider now a point $a \in \Sigma^{\prime \prime} \backslash \Sigma^{\prime}$. Since the function $\nu\left(S_{0}, a\right)$ is upper semi-continuous in $a$ with respect to the Zariski topology, it is enough to check that $\nu\left(S_{0}, a\right)$ is positive at generic points $a \in$ $\Sigma^{\prime \prime} \backslash \Sigma^{\prime}$. We then choose $c_{1}$ large enough in order to get Lelong numbers larger than 1 . So we can assume that $a$ is a regular point of $\Sigma^{\prime \prime} \backslash \Sigma^{\prime}$ and there is a point $\widehat{a} \in \tau_{2}^{-1}(a)$ such that $\tau_{2}^{-1}\left(\Sigma^{\prime \prime}\right)$ is a hypersurface smooth at $\widehat{a}$.

Choose local coordinates $\widehat{z}=\left(\widehat{z}_{1}, \ldots, \widehat{z}_{k}\right)$ on a neighbourhood of $\widehat{a}$ such that $\widehat{z}=0$ at $\widehat{a}$ and $\tau_{2}^{-1}\left(\Sigma^{\prime \prime}\right)$ is given by $\widehat{z}_{1}=0$. Since $\Sigma^{\prime \prime}$ has codimension $\geq 2$, we can choose $\widehat{z}$ so that the hyperplanes $\widehat{H}_{\xi}:=\left\{\widehat{z}_{k}=\xi\right\}$ parallel to $\left\{\widehat{z}_{k}=0\right\}$ are sent to hypersurfaces, denoted by $H_{\xi}$, which contain $\Sigma^{\prime \prime}$ in a neighbourhood of $a$. 
The average of $\left[\widehat{H}_{\xi}\right]$ with respect to the Lebesgue measure on $\xi$ is a smooth form $\widehat{\Theta}$. So it is bounded by a constant times $\widehat{\omega}$. On the other hand, since $\left[H_{\xi}\right]$ has positive Lelong number at $a,\left(\tau_{2}\right)_{*}(\widehat{\Theta})$ has positive Lelong number at $a$. We conclude that $\left(\tau_{2}\right)_{*}(\widehat{\omega})$ has positive Lelong number at $a$. This completes the proof of the lemma.

We show that $R$ satisfies Proposition 3.1. Fix a point $a$ in $X$ such that $\nu(R, a) \leq \nu$. Fix also local holomorphic coordinates near $a$. We will first construct inverse branches for flat holomorphic discs through $a$ and then extend these inverse branches to a small ball centered at $a$. We will identify a neighbourhood of $a$ to the unit ball in $\mathbb{C}^{k}$ for simplicity. The following version of Sibony-Wong's theorem is the tool for this extension.

Let $\mathbb{B}_{r}$ denote the ball of center 0 and of radius $r$ in $\mathbb{C}^{k}$. The family $\mathscr{F}$ of complex lines through 0 is parametrized by the projective space $\mathbb{P}^{k-1}$ which is endowed with the natural Fubini-Study metric. This metric induces a natural probability measure on $\mathscr{F}$ that we denote by $\mathscr{L}$. If $\Delta$ is an element of $\mathscr{F}$, denote by $\Delta_{r}$ its intersection with $\mathbb{B}_{r}$.

Proposition 3.3. Let $0<\delta_{0} \leq 1$ be a constant. Let $\mathscr{F}^{\prime} \subset \mathscr{F}$ be such that $\mathscr{L}\left(\mathscr{F}^{\prime}\right) \geq \delta_{0}$, and $A$ the intersection of $\mathscr{F}^{\prime}$ with $\mathbb{B}_{r}$. Let $h: A \rightarrow \mathbb{C}^{l}$ be a map which is holomorphic on each $\Delta_{r}$ for $\Delta \in \mathscr{F}^{\prime}$ and which can be extended holomorphically to a neighbourhood of 0 . Then $h$ can be extended to a holomorphic map from $\mathbb{B}_{\lambda r}$ to $\mathbb{C}^{l}$, where $0<\lambda \leq 1$ is a constant depending on $\delta_{0}$ but independent of $l, \mathscr{F} \mathscr{F}^{\prime}$ and $r$. Moreover, if the extension is still denoted by $h$ then

$$
\sup _{\mathbb{B}_{\lambda r}}\|h-h(0)\| \leq \sup _{A}\|h-h(0)\| .
$$

In particular, if $\|h-h(0)\|<\rho$ and if $h(A)$ does not intersect a complex hypersurface $Z$ of the ball of center $h(0)$ and radius $\rho$, then $h\left(\mathbb{B}_{\lambda r}\right)$ satisfies the same property.

Proof. When $l=1$ the result is due to Sibony-Wong [25]. We easily deduce from their result the holomorphic extension of $h$ for any dimension $l$. In order to get the inequality in the proposition, assume $h(0)=0$ for simplicity. Let $z$ be a point in $\mathbb{B}(0, \lambda r)$ we have to show that $\|h(z)\| \leq \sup _{A}\|h\|$. Composing $h$ with a rotation on $\mathbb{C}^{l}$ allows to assume that $h(z)=(\|h(z)\|, 0, \ldots, 0)$. We obtain the desired inequality by using Sibony-Wong's theorem for the first coordinate function of $h$.

For the last assertion, we can write $Z=\{g=0\}$ where $g$ is a holomorphic function on the ball of center $h(0)$ and of radius $\rho$. Sibony-Wong's theorem applied to $1 / g \circ h$ implies that $1 / g \circ h$ is holomorphic on $\mathbb{B}_{\lambda r}$. Hence $h\left(\mathbb{B}_{\lambda r}\right)$ does not intersect $Z$. The proposition follows. 
In order to control the size of holomorphic discs, we need the following lemma, see [14, Lemma 1.55] for the proof which is valid for any compact complex manifold $Y$.

Lemma 3.4. Let $Y$ be a compact complex manifold endowed with a fixed Hermitian metric. Let $\delta_{1}>0$ be a constant small enough depending on $Y$. Let $g: \Delta_{r} \rightarrow Y$ be a holomorphic map from the disc of center 0 and of radius $r$ in $\mathbb{C}$ to $Y$. Assume that the area of $g\left(\Delta_{r}\right)$, counted with multiplicity, is smaller than $\delta_{1}$. Then for any $\epsilon>0$, there is a constant $0<\lambda<1$ independent of $g$ and $r$ such that the diameter of $g\left(\Delta_{\lambda r}\right)$ is at most equal to $\epsilon \sqrt{\operatorname{area}\left(g\left(\Delta_{r}\right)\right)}$.

We will apply it to $Y=\widehat{\Gamma}$. So from now on we fix a constant $\delta_{1}$ satisfying the last lemma for $\widehat{\Gamma}$.

Note that the current $R$ constructed above can be seen as the obstruction to the existence of good inverse branches for balls in the spirit of Proposition 3.1. In order to measure the obstruction to the inverse branches of discs through a point $a$ we have to slice this current using complex lines through $a$. We will need the following known technical result, see Lemma 5.52 in [14]. Recall that we identify a neighbourhood of $a$ in $X$ to the unit ball in $\mathbb{C}^{k}$ for simplicity.

Lemma 3.5. Let $T$ be a positive closed $(1,1)$-current on $X$. Then for any constant $0<\delta_{2}<1$ there is a constant $r>0$ and a family $\mathscr{F}^{\prime} \subset \mathscr{F}$, such that $\mathscr{L}\left(\mathscr{F}^{\prime}\right) \geq 1-\delta_{2}$, and for every $\Delta \in \mathscr{F}^{\prime}$ the measure $T \wedge\left[\Delta_{r}\right]$ is well-defined and of mass smaller than or equal to $\nu(T, a)+\delta_{2}$. Here, $\nu(T, a)$ denotes the Lelong number of $T$ at $a$.

Recall that locally we can write $T=d d^{c} u$ with $u$ a p.s.h. function. The measure $T \wedge\left[\Delta_{r}\right]$ is well-defined if $u$ is not identically $-\infty$ on $\Delta_{r}$. This property holds for $\mathscr{L}$-almost every $\Delta$ and we have $T \wedge\left[\Delta_{r}\right]:=d d^{c}\left(u\left[\Delta_{r}\right]\right)$.

We are now ready to construct inverse branches for discs through the point $a$ under the hypotheses of Proposition 3.1. Fix a value of the constant $c_{1} \geq \delta_{1}^{-1}$ in the definition of $S$ satisfying Lemma 3.2. We have the following lemma.

Lemma 3.6. There is a number $r_{0}>0$ and a family $\mathscr{F}_{0} \subset \mathscr{F}$ with $\mathscr{L}\left(\mathscr{F}_{0}\right) \geq$ $1-\nu / 2$ satisfying the following property. For every complex line $\Delta \in \mathscr{F}_{0}$ and for every $n \geq 0$, the disc $\Delta_{r_{0}}$ admits at least $(1-\nu / 2) d_{t}^{n}$ inverse branches $g: \Delta_{r_{0}} \rightarrow$ $\Delta_{r_{0},-n}$ of order $n$ such that if we define $\Delta_{r_{0},-i}:=f^{n-i}\left(\Delta_{r_{0},-n}\right)$ for $0 \leq i \leq n$ and $\widehat{\Delta}_{r_{0},-i}:=\tau_{1}^{-1}\left(\Delta_{r_{0},-i-1}\right)$ for $0 \leq i \leq n-1$, then $\Delta_{r_{0},-i} \cap \Sigma=\varnothing$ for $0 \leq i \leq n$ and the diameters of $\widehat{\Delta}_{r_{0},-i}$ for $0 \leq i \leq n-1$ are smaller than $\frac{1}{2} \theta^{i / 2}$.

Note that since $\Delta_{r_{0},-i} \cap \Sigma=\varnothing$ for $0 \leq i \leq n, \tau_{1}$ defines a biholomorphic map between $\widehat{\Delta}_{r_{0},-i}$ and $\Delta_{r_{0},-i-1}$ and $\tau_{2}$ defines a biholomorphic map between $\widehat{\Delta}_{r_{0},-i}$ and $\Delta_{r_{0},-i}$. Moreover, since $\widehat{\omega} \geq \tau_{1}^{*}(\omega)$ and $\widehat{\omega} \geq \tau_{2}^{*}(\omega)$, the diameter of $\widehat{\Delta}_{r_{0},-i}$ is larger than or equal to the diameters of $\Delta_{r_{0},-i-1}$ and of $\Delta_{r_{0},-i}$. So the diameter of $\Delta_{r_{0},-i}$ is smaller than $\frac{1}{2} \theta^{i / 2}$ for $0 \leq i \leq n$. 
Proof. Observe that if $g$ is an inverse branch of order $n$ satisfying the properties in the lemma then $f \circ g$ is an inverse branch of order $n-1$ satisfying the same properties. So we only have to prove the lemma for $n=m N$ where $m$ is an integer.

By Lemma 3.4, we only need to bound the area of $\widehat{\Delta}_{r_{0},-i}$ by $\theta^{i} / c_{1} \leq \delta_{1}$ and then reduce the radius $r_{0}$ in order to get the diameter control. The rest of the lemma is obtained by induction on $m$. We will only consider discs $\Delta_{r}$ through $a$ which are not contained in the orbit of $\Sigma$. This property holds for almost every disc.

By Lemma 3.5 applied to $R$ and to $\delta_{2}:=\nu / 2$, we can choose a number $r$ and a family $\mathscr{F}_{0} \subset \mathscr{F}$ with $\mathscr{L}\left(\mathscr{F}_{0}\right) \geq 1-\nu / 2$ such that for $\Delta \in \mathscr{F}_{0}$ the measure $R \wedge\left[\Delta_{r}\right]$ is well-defined and of mass smaller than $2 \nu$. Let $\nu_{m}$ denote the mass of $d_{t}^{-N m}\left(f^{N}\right)_{*}^{m}(S) \wedge\left[\Delta_{r}\right]$. By definition of $R$, we have $\sum_{m>0} \theta^{-N m} \nu_{m} \leq \nu / 4$.

We show by induction on $m$ that for every $\Delta \in \mathscr{F}_{0}$ the disc $\Delta_{r}$ admits at least $\gamma_{m}:=\left(1-2 \sum_{0 \leq i<m} \theta^{-N i} \nu_{i}\right) d_{t}^{N m}$ inverse branches $g_{-n}^{(s)}: \Delta_{r} \rightarrow \Delta_{r,-N m}^{(s)}$ of order $N m$ such that the area of $\widehat{\Delta}_{r,-i}^{(s)}$ is smaller than $\theta^{i} / c_{1}$ and $\Delta_{r,-i}^{(s)} \cap \Sigma=\varnothing$ for $0 \leq i \leq N m$. We used here similar notation as the one introduced in the statement of the lemma. The index $s$ satisfies $1 \leq s \leq s_{m}$ for some integer $s_{m}$ with $\gamma_{m} \leq s_{m} \leq d_{t}^{N m}$. Then the above discussion implies the result. Assume this property for $m$. The case $m=0$ is clear since the choice of $\mathscr{F}_{0}$ implies that $\Delta_{r}$ is out of $\Sigma$. We construct inverse branches of order $N(m+1)$.

The property $\Delta_{r,-N m}^{(s)} \cap \Sigma=\varnothing$ and the definition of $\Sigma$ allow us to define the maximal number $d_{t}^{N}$ inverse branches of order $N$ for each $\Delta_{r,-N m}^{(s)}$. Composing them with the inverse branches of order $N m$ of $\Delta$ gives $\gamma_{m} d_{t}^{N}$ inverse branches of order $N(m+1)$ for $\Delta$. We will count and remove the ones which do not satisfy the area control. We call them large-sized inverse branches. We also have to remove later inverse branches whose images intersect $\Sigma$. We first count the number of large-sized inverse branches of order $N$ of $\Delta_{r,-N m}^{(s)}$ for each $s$. For simplicity, we will drop the letter $s$ for the moment.

Consider all inverse branches $g: \Delta_{r,-N m} \rightarrow \Delta_{r,-N m-n}$ of order $1 \leq n \leq N$ of $\Delta_{r,-N m}$ such that the area of $\widehat{\Delta}_{r,-N m-i}$ is bounded by $\theta^{N m+i} / c_{1}$ for $i \leq n-1$ but not for $i=n$. They are completely disjoint in the sense that such two different branches are not extensions of the same branch of lower order of $\Delta_{r,-N m}$. The extensions of order $N(m+1)$ of these branches are exactly the large-sized ones. So the number of large-sized branches of order $N(m+1)$ extending $g$ is $d_{t}^{N-n}$.

Observe that the area of $\widehat{\Delta}_{r,-N m-n}$ is the mass of $\left[\Delta_{r,-N m-n}\right] \wedge\left(\tau_{2}\right)_{*}(\widehat{\omega})$. This mass is smaller than or equal to the mass of $\left[\Delta_{r,-N m}\right] \wedge\left(f_{*}\right)^{n}\left(\tau_{2}\right)_{*}(\widehat{\omega})$ because $f^{n}$ defines a biholomorphic map from a neighbourhood of $\Delta_{r,-N m-n}$ to a neighbourhood of $\Delta_{r,-N m}$. So the sum of these areas over all these branches $g$ (there are at most $d_{t}^{N}$ such maps) is bounded by $c_{1}^{-1} \theta^{N}$ times the mass of $S \wedge\left[\Delta_{r,-N m}\right]$. Since the area of $\widehat{\Delta}_{r,-N m-n}$ is larger than $\theta^{N m+n} / c_{1}$, the number of considered maps $g$ is at most equal to $\theta^{-N m}$ times the mass of $S \wedge\left[\Delta_{r,-N m}\right]$. The number of 
large-sized inverse branches of order $N$ of $\Delta_{r,-N m}$ to remove is at most equal to $\theta^{-N m} d_{t}^{N}$ times the mass of $S \wedge\left[\Delta_{r,-N m}\right]$.

Now, the number $M$ of all large-sized inverse branches of order $N(m+1)$ of $\Delta_{r}$ to remove is bounded by $\theta^{-N m} d_{t}^{N}$ times the mass of $\sum_{s} S \wedge\left[\Delta_{r,-N m}^{(s)}\right]$. By the definition of inverse branches, the last mass is bounded by the one of $\left(f^{N}\right)_{*}^{m}(S) \wedge\left[\Delta_{r}\right]$ which is equal to $d_{t}^{N m} \nu_{m}$. We conclude that $M \leq \theta^{-N m} d_{t}^{N(m+1)} \nu_{m}$. Therefore, the number of inverse branches of order $N(m+1)$ satisfying the area control is larger than or equal to $\gamma_{m} d_{t}^{N}-M \geq \gamma_{m+1}+\nu_{m} d_{t}^{N(m+1)}$.

It remains to remove the inverse branches whose images intersect $\Sigma$. Denote by $t_{m+1}$ the number of inverse branches $g: \Delta_{r} \rightarrow \Delta_{r,-N(m+1)}$ of order $N(m+1)$ such that $\Delta_{r,-N m-i} \cap \Sigma \neq \varnothing$ for some $1 \leq i \leq N$. By Lemma 3.2, the intersection of $\left[\Delta_{r,-N m-i}\right]$ with the current $S_{0}$ is a positive measure of mass at least equal to 1.

By definition of inverse branches, the map $f^{n}$ is holomorphic and injective on a neighbourhood of $\Delta_{r,-n}$ with image in a neighbourhood of $\Delta_{r}$ for every $n \leq N(m+1)$. We then deduce that the mass of $\left(f^{N}\right)_{*}^{m}(S) \wedge\left[\Delta_{r}\right]$ is at least equal to $t_{m+1}$. It follows that $t_{m+1} \leq \nu_{m} d_{t}^{N m}$. We conclude that the number of inverse branches of order $N(m+1)$ satisfying the properties in the lemma is at least equal to $\gamma_{m+1}$. This completes the proof of the lemma.

End of the proof of Proposition 3.1. We now apply Lemma 3.6 and Proposition 3.3 for $\delta_{0}=\nu / 4$. Let $a_{-n}^{(1)}, \ldots, a_{-n}^{(s)}$ with $0 \leq s \leq d_{t}^{n}$ be the distinct points in $f^{-n}(a)$ such that $f^{i}\left(a_{-n}^{(j)}\right) \notin \Sigma$ for all $0 \leq i \leq n$ and $1 \leq j \leq s$. If $g: \Delta_{r_{0}} \rightarrow \Delta_{r_{0},-n}$ is an inverse branch as in Lemma 3.6, then $\Delta_{r_{0},-n}$ contains exactly one of the points $a_{-n}^{(j)}$. We say that $a_{-n}^{(j)}$ is the center of $\Delta_{r_{0},-n}$.

Denote by $\mathscr{F}^{(j)}$ the set of $\Delta \in \mathscr{F}$ such that $\Delta_{r_{0}}$ admits an inverse branch of order $n$ as in Lemma 3.6 with center $a_{-n}^{(j)}$. Let $S_{n}$ denote the set of all $j$ such that $\mathscr{L}\left(\mathscr{F}^{(j)}\right) \geq \nu / 4$. Let $j$ be an element of $S_{n}$. We show that $B(a, r)$ admits an inverse branch of order $n$ of size $\leq \theta^{n / 2}$ with center $a_{-n}^{(j)}$ for a suitable constant $r>0$ independent of $n$.

Let $A^{(j)}$ denote the intersection of $\mathscr{F}^{(j)}$ with $B\left(a, r_{0}\right)$. The inverse branches of $\Delta_{r_{0}}$ with $\Delta \in \mathscr{F}^{(j)}$ with images centered at $a_{-n}^{(j)}$ agree at the common intersection point $a$ and form a map $g: A^{(j)} \rightarrow A_{-n}^{(j)}$ where $A_{-n}^{(j)}$ is the union of $\Delta_{r_{0},-n}$ centered at $a_{-n}^{(j)}$ with $\Delta \in \mathscr{F}^{(j)}$. Define as above $a_{-i}^{(j)}:=f^{n-i}\left(a_{-n}^{(j)}\right), A_{-i}^{(j)}:=f^{n-i}\left(A_{-n}^{(j)}\right)$ for $0 \leq i \leq n$ and $\widehat{a}_{-i}^{(j)}:=\tau_{1}^{-1}\left(a_{-i-1}^{(j)}\right), \widehat{A}_{-i}^{(j)}:=\tau_{1}^{-1}\left(A_{-i-1}^{(j)}\right)$ for $0 \leq i \leq n-1$.

By Lemma 3.6, we have $A_{-i}^{(j)} \cap \Sigma=\varnothing$ for $0 \leq i \leq n$. Therefore, the map $\tau_{1}^{-1} \circ f^{n-i-1} \circ g$ extends holomorphically to a neighbourhood of $A^{(j)}$. Moreover, the image of $A^{(j)}$ is equal to $\widehat{A}_{-i}^{(j)}$ which is contained in the ball of radius $\frac{1}{2} \theta^{i / 2} \leq 1$ centered at $\widehat{a}_{-i}^{(j)}$ and does not intersect the hypersurface $\tau_{1}^{-1}(\Sigma) \cup \tau_{2}^{-1}(\Sigma)$. Recall that the metric $\widehat{\omega}$ on $\widehat{\Gamma}$ is chosen so that any ball of radius 1 is contained in an open set biholomorphic to a ball in $\mathbb{C}^{k}$. So Proposition 3.3 can be applied to 
this map. According to that proposition, for $r$ small enough (equal to $r_{0}$ times a constant independent of $n, i, j)$, all maps $f^{n-i} \circ g$ and $\tau_{1}^{-1} \circ f^{n-i-1} \circ g$ extend holomorphically to $B(a, r)$. Moreover, their images, denoted by $B(a, r)_{-i}^{(j)}$ and $\widehat{B}(a, r)_{-i}^{(j)}$ respectively, have diameters smaller than or equal to $\theta^{i / 2}$. We also have $\widehat{B}(a, r)_{-i}^{(j)} \cap \tau_{1}^{-1}(\Sigma)=\varnothing$ and $\widehat{B}(a, r)_{-i}^{(j)} \cap \tau_{2}^{-1}(\Sigma)=\varnothing$ for $0 \leq i \leq n-1$. It follows that $B(a, r)_{-i}^{(j)} \cap \Sigma=\varnothing$ for $0 \leq i \leq n$. So the extension of $g$ defines an inverse branch of order $n$ and of size $\leq \theta^{n / 2}$ on $B(a, r)$.

It remains now to show that $S_{n}$ contains at least $(1-\nu) d_{t}^{n}$ elements. By Lemma 3.6, we have $\sum_{j} \mathscr{L}\left(\mathscr{F}^{(j)}\right) \geq d_{t}^{n}(1-\nu / 2)^{2}$. Since $\mathscr{L}\left(\mathscr{F}^{(j)}\right) \leq \mathscr{L}(\mathscr{F})=1$, we deduce that the last sum is bounded by $\# S_{n}+\left(d_{t}^{n}-\# S_{n}\right) \nu / 4$. It follows that $\# S_{n}+\left(d_{t}^{n}-\# S_{n}\right) \nu / 4 \geq d_{t}^{n}(1-\nu / 2)^{2}$. Hence, $\# S_{n} \geq(1-\nu) d_{t}^{n}$. This completes the proof of the proposition.

\section{Exceptional set for backward orbits}

In this section, we give the proof of Theorem 1.2. In what follows, we only consider points $a$ outside $I_{\infty} \cup I_{\infty}^{\prime}$. We need the following result that was obtained in [13] in a more general setting.

Lemma 4.1. There is a pluripolar subset $E$ of $X$ containing $I_{\infty}^{\prime}$ such that if $a$ is out of $E$ then $\mu_{n}^{a}$ converges to $\mu$ as $n$ goes to infinity.

For every $a \notin I_{\infty}^{\prime}$, define $\epsilon_{a}:=\sup \left\|\mu^{a}-\mu\right\|$, where the supremum is taken over all cluster values $\mu^{a}$ of the sequence $\mu_{n}^{a}$. So we have $\mu_{n}^{a} \rightarrow \mu$ if and only if $\epsilon_{a}=0$. We deduce from the above lemma and Proposition 3.1 the following property.

Lemma 4.2. Let a be a point out of $I_{\infty}^{\prime}$. Then, we have $\epsilon_{a} \leq 2 \nu(R, a)$. In particular, $\mu_{n}^{a} \rightarrow \mu$ if $\nu(R, a)=0$.

Proof. We have seen that the condition $a \notin I_{\infty}^{\prime}$ is necessary to define $\mu_{n}^{a}$. Since we always have $\epsilon_{a} \leq 2$, we only need to consider the case where $\nu(R, a)<1$. Fix a constant $\nu(R, a)<\nu \leq 1$. Let $B(a, r)$ be a ball as in the conclusion of Proposition 3.1. Choose also a point $b$ in $B(a, r) \backslash E$. Such a choice is always possible since $E$ is pluripolar. Write

$$
f^{-n}(a)=a_{-n}^{(1)}, \ldots, a_{-n}^{\left(d_{t}^{n}\right)}
$$

and

$$
f^{-n}(b)=b_{-n}^{(1)}, \ldots, b_{-n}^{\left(d_{t}^{n}\right)}
$$

where each points are repeated according to its multiplicity.

Proposition 3.1 implies that we can arrange these points so that the distance between $a_{-n}^{(j)}$ and $b_{-n}^{(j)}$ is smaller than $\left(d_{k-1} / d_{t}+\epsilon\right)^{n / 2}$ for at least $(1-\nu) d_{t}^{n}$ indices $j$. Here $\epsilon>0$ is a fixed constant small enough. Since $\left(d_{k-1} / d_{t}+\epsilon\right)^{n / 2}$ tends to 0 , 
we then deduce that any cluster values of the sequence $\mu_{n}^{a}-\mu_{n}^{b}$ is a measure of mass at most equal to $2 \nu$. The property holds for every $\nu>\nu(R, a)$. Hence, the result follows from Lemma 4.1 which implies that $\mu_{n}^{b} \rightarrow \mu$.

The exceptional set in Theorem 1.2 is given in the following proposition.

Proposition 4.3. There is a proper analytic subset $\mathscr{E}$ of $X$, possibly empty, satisfying the following three conditions: (1) no component of $\mathscr{E}$ is contained in $I_{\infty} \cup I_{\infty}^{\prime}$; (2) $f^{-1}\left(\mathscr{E} \backslash I^{\prime}\right) \subset \mathscr{E}$; (3) any proper analytic subset of $X$ satisfying (1) and (2) is contained in $\mathscr{E}$. Moreover, we have

$$
\mathscr{E}=\overline{f^{-1}\left(\mathscr{E} \backslash I^{\prime}\right)}=\overline{f(\mathscr{E} \backslash I)} .
$$

Proof. Consider the set $Y_{0}:=\{\nu(R, a) \geq 1\}$. By Siu's theorem, $Y_{0}$ is a proper analytic subset of $X$ [26]. Denote for $n \geq 1$ the analytic set $Y_{n}$ which is the closure of the set

$$
\left\{z \notin I_{\infty} \cup I_{\infty}^{\prime}, \quad f^{-i}(z) \in Y \text { for } 0 \leq i \leq n\right\}
$$

Since the sequence $Y_{n}$ is decreasing, it is stationary: we have $Y_{n}=Y_{n+1}$ for $n$ large enough. Denote by $\mathscr{E}:=Y_{n}$ for $n$ large enough.

It is clear that $\mathscr{E}$ satisfies the property (1). We have by definition

$$
\overline{f^{-1}\left(\mathscr{E} \backslash\left(I_{\infty} \cup I_{\infty}^{\prime}\right)\right)} \subset \mathscr{E}
$$

Since $f^{-1}\left(\mathscr{E} \backslash\left(I_{\infty} \cup I_{\infty}^{\prime}\right)\right)$ is dense in $f^{-1}\left(\mathscr{E} \backslash I^{\prime}\right)$, the set $\mathscr{E}$ satisfies (2). Denote by $\mathscr{E}_{n}$ the closure of $f^{-n}\left(\mathscr{E} \backslash\left(I_{\infty} \cup I_{\infty}^{\prime}\right)\right)$. This is a decreasing sequence of analytic sets satisfying the property (1). So it is stationary: we have $\mathscr{E}_{n}=\mathscr{E}_{n+1}$ for $m$ large enough. Since $f\left(\mathscr{E}_{n+1} \backslash I\right)$ is dense in $\mathscr{E}_{n}$, we deduce from the last identity that $\mathscr{E}_{n-1}=\mathscr{E}_{n}$ and hence, by induction, $\mathscr{E}_{1}=\mathscr{E}$. It follows that $\mathscr{E}=\overline{f^{-1}\left(\mathscr{E} \backslash I^{\prime}\right)}$ which also implies that $\mathscr{E}=\overline{f(\mathscr{E} \backslash I)}$.

Let $\mathscr{E}^{\prime}$ be a proper analytic subset of $X$ satisfying (1) and (2). We have to show that $\mathscr{E}^{\prime} \subset \mathscr{E}$. Property (2) implies that if $a$ is a point in $\mathscr{E}^{\prime} \backslash I_{\infty}^{\prime}$ then any cluster values of $\mu_{n}^{a}$ is supported by $\mathscr{E} \prime$. Since $\mu$ has no mass on $\mathscr{E}^{\prime}$, we deduce that $\epsilon_{a}=2$. Lemma 4.2 implies that $a$ is in $Y_{0}$. So we have $\mathscr{E}^{\prime} \subset Y_{0}$. Property (2) again, together with the definition of $\mathscr{E}$, implies that $\mathscr{E}^{\prime} \subset \mathscr{E}$. This completes the proof of the proposition.

We need the following characterization of the exceptional set $\mathscr{E}$.

Lemma 4.4. Let $Y$ be a proper analytic subset of $X$. Let a be a point in $Y$ which does not belong to $I_{\infty} \cup I_{\infty}^{\prime}$. Let $\lambda_{n}(a)$ denote the number of backward orbits $a_{0}, a_{-1}, \ldots, a_{-n}$ counted with multiplicity with $a_{0}=a, a_{-i-1} \in f^{-1}\left(a_{-i}\right)$ for $0 \leq i \leq n-1$ and $a_{-i} \in Y$ for $0 \leq i \leq n$. If a is not in $\mathscr{E}$ then $d_{t}^{-n} \lambda_{n}(a) \rightarrow 0$ as $n \rightarrow \infty$. 
Proof. Observe that since $a$ is out of $I_{\infty} \cup I_{\infty}^{\prime}$ the same property holds for $a_{-i}$. By definition, the sequence $d_{t}^{-n} \lambda_{n}(a)$ is decreasing because each backward orbit of order $n+1$ is one of the $d_{t}$ extensions of backward orbits of order $n$. Since the functions $\lambda_{n}$ are upper-semi-continuous with respect to the induced Zariski topology on $Y \backslash\left(I_{\infty} \cup I_{\infty}^{\prime}\right)$, the function $\lambda:=\lim d_{t}^{-n} \lambda_{n}$ is also upper semicontinuous with respect to this topology. Let $m$ denote the maximal value of $\lambda$ on $Y \backslash\left(I_{\infty} \cup I_{\infty}^{\prime} \cup \mathscr{E}\right)$. It is enough to show that $m=0$.

Assume that $m>0$. Denote by $Z^{*}$ the set of points $a \in Y \backslash\left(I_{\infty} \cup I_{\infty}^{\prime} \cup \mathscr{E}\right)$ such that $\lambda(a) \geq m$. The closure $Z$ of $Z^{*}$ is an analytic subset of $Y$. No irreducible component of $Z$ is contained in $\mathscr{E}$. Consider a point $a \in Z^{*}$. The invariance properties of $\mathscr{E}$ imply that $f^{-1}(a) \cap \mathscr{E}=\varnothing$. Using the definition of $\lambda$ and of $m$, we have

$$
m=\lambda(a)=d_{t}^{-1} \sum_{b \in f^{-1}(a) \cap Y} \lambda(b) .
$$

Since $\lambda(b) \leq m$ and $\# f^{-1}(a)=d_{t}$, we deduce that $f^{-1}(a) \subset Z$ and $\lambda(b)=m$ for $b \in f^{-1}(a)$. Therefore, $f^{-1}\left(Z^{*}\right) \subset Z$ and $f^{-1}\left(Z \backslash I^{\prime}\right) \subset Z$ since $f^{-1}\left(Z^{*}\right)$ is dense in $f^{-1}\left(Z \backslash I^{\prime}\right)$. Proposition 4.3 implies that $Z \subset \mathscr{E}$. This is a contradiction. The lemma follows.

End of the proof of Theorem 1.2. Let $a$ be a point out of $I_{\infty} \cup I_{\infty}^{\prime}$. If $a$ is in $\mathscr{E}$, it is clear that $\mu_{n}^{a}$ does not converge to $\mu$. So assume that $a \notin \mathscr{E}$. We have to show that $\epsilon_{a}=0$. Fix a constant $\nu>0$. It is enough to prove that $\epsilon_{a} \leq 4 \nu$. Define $Y:=\{\nu(R, \cdot) \geq \nu\}$. By Siu's theorem, this is a proper analytic subset of $X$. By Lemma 4.2, the case where $a \notin Y$ is clear. From now on assume that $a \in Y$. By Lemma 4.4 applied to $Y$, we have $\lambda_{m}(a) \leq \nu d_{t}^{m}$ for some integer $m$ large enough.

Consider all backward orbits of $a$ of order $l \leq m$ of the form

$$
\mathscr{O}:=\left\{a_{0}, a_{-1}, \ldots, a_{-l}\right\} \quad \text { with } a_{0}=a, f\left(a_{-i-1}\right)=a_{-i} \text { for } 0 \leq i \leq l-1
$$

such that $a_{-i} \in Y$ for $i \leq l-1$ and $a_{-l} \notin Y$ unless $l=m$. These orbits are counted with multiplicity. Using that $\mu_{n}^{a}$ is the probability measure equidistributed on $f^{-n}(a)$, it is not difficult to see that

$$
\mu_{n}^{a}=\sum_{\mathscr{O}} d_{t}^{-l} \mu_{n-l}^{a_{-}}
$$

for every $n \geq m$. By considering the masses of the measures in the above identity, we have

$$
\sum_{\mathscr{O}} d_{t}^{-l}=1
$$

We then deduce from the same identity that

$$
\epsilon_{a} \leq \sum_{\mathscr{O}} d_{t}^{-l} \epsilon_{a_{-l}} .
$$


Let $\Sigma, \Sigma^{\prime}$ denote the sets of $\mathscr{O}$ with $a_{-l} \in Y$ (hence $l=m$ ) and with $a_{-l} \notin Y$ respectively. By definition of $\lambda_{n}$, we have

$$
\sum_{\mathscr{O} \in \Sigma} d_{t}^{-l}=d_{t}^{-m} \lambda_{m}(a) \leq \nu
$$

On the other hand, we have $\epsilon_{a_{-l}} \leq 2$ for every $\mathscr{O}$ and by Lemma 4.2, $\epsilon_{a_{-l}} \leq 2 \nu$ for $\mathscr{O} \in \Sigma^{\prime}$. It follows that

$$
\epsilon_{a} \leq \sum_{\mathscr{O} \in \Sigma} d_{t}^{-l} \epsilon_{a_{-l}}+\sum_{\mathscr{O} \in \Sigma^{\prime}} d_{t}^{-l} \epsilon_{a_{-l}} \leq 2 \sum_{\mathscr{O} \in \Sigma} d_{t}^{-l}+2 \nu \sum_{\mathscr{O} \in \Sigma^{\prime}} d_{t}^{-l} \leq 2 \nu+2 \nu=4 \nu .
$$

This completes the proof of the theorem.

Remark 4.5. Define by induction $\mathscr{E}_{0}:=\mathscr{E}, \mathscr{E}_{n}:=f\left(\mathscr{E}_{n-1}\right)$ and $\mathscr{E}_{\infty}:=\cup_{n \geq 0} \mathscr{E}_{n}$. If $a$ is a point in $\mathscr{E}_{\infty} \backslash I_{\infty}^{\prime}$, then $\mu_{n}^{a}$ has positive mass on $\mathscr{E}$ for some $n \geq 0$. It follows from the invariance properties of $\mathscr{E}$ that $\mu_{n}^{a}$ does not converge to $\mu$ since $\mu$ has no mass on $\mathscr{E}$. Lemma 4.4 still holds for $a \notin \mathscr{E}_{\infty} \cup I_{\infty}^{\prime}$. We can show that $\mu_{n}^{a} \rightarrow \mu$ for such points $a$. This property is slightly stronger than Theorem 1.2.

\section{$5 \quad$ Periodic points and Lyapounov exponents}

In this section, we give the proof of Theorem 1.1 and then a lower bound for the Lyapounov exponents of the equilibrium measure.

We call fixed point of $f$ any point $a$ such that $(a, a)$ belongs to the closure $\Gamma$ of the graph of $f$ in $X \times X$. A fixed point $a$ is isolated if $(a, a)$ is isolated in the intersection of $\Gamma$ with the diagonal $\Delta$ of $X \times X$. The multiplicity of an isolated periodic point $a$ is the multiplicity of the intersection $\Gamma \cap \Delta$ at $(a, a)$. A fixed point $a$ is regular if it is not an indeterminacy point, that is, $a \notin I$. Such a point is called repelling if all the eigenvalues of the differential of $f$ at $a$ have modulus strictly larger than 1 . So repelling fixed points are isolated with multiplicity 1.

Periodic points of period $n$ are fixed points of $f^{n}$. A periodic point $a$ of order $n$ is regular if $f^{i}(a) \notin I$ for every $i \in \mathbb{N}$. Such a point is said to be repelling if it is moreover a repelling fixed point of $f^{n}$. We need the following upper bound for the number of isolated periodic points.

Proposition 5.1. Let $P_{n}$ denote the number of isolated periodic points of period $n$ of $f$. Then $\# P_{n} \leq d_{t}^{n}+o\left(d_{t}^{n}\right)$ as $n$ goes to infinity.

We first prove the following property.

Lemma 5.2. Let $\Gamma_{n}$ denote the closure of the graph of $f^{n}$ in $X \times X$. Then the sequence of positive closed $(k, k)$-currents $d_{t}^{-n}\left[\Gamma_{n}\right]$ converges to $\pi_{1}^{*}(\mu)$ as $n$ goes to infinity. Here, $\pi_{1}: X \times X \rightarrow X$ is the natural projection onto the first factor. 
Proof. Denote by $z=\left(z_{1}, z_{2}\right)$ a general point in $X \times X$ with $z_{1}, z_{2} \in X$. Observe that smooth $(k, k)$-forms on $X \times X$ can be written as a finite combination of forms of types

$$
\Phi(z):=u(z) \Omega\left(z_{1}\right) \wedge \alpha\left(z_{1}\right) \wedge \Theta\left(z_{2}\right) \wedge \beta\left(z_{2}\right),
$$

where $u$ is a smooth function, $\Omega, \Theta$ are smooth positive forms and $\alpha, \beta$ are smooth forms of bidegree $(p, 0)$ or $(0, p)$ for some $p \geq 0$. We have to check that

$$
\left\langle d_{t}^{-n}\left[\Gamma_{n}\right]-\pi_{1}^{*}(\mu), \Phi\right\rangle \rightarrow 0 .
$$

Case 1. Assume that $\Omega$ is a function, $\Theta$ is of bidegree maximal $(k, k)$ and $p=0$. We may, for simplicity, assume that $\Omega=1, \alpha=1$ and $\beta=1$. We have by Fubini's theorem

$$
\left\langle d_{t}^{-n}\left[\Gamma_{n}\right], \Phi\right\rangle=\int_{a \in X} d_{t}^{-n} \sum_{b \in f^{-1}(a)} u(b, a) \Theta(a)=\int_{a \in X}\left\langle\mu_{n}^{a}, u(\cdot, a)\right\rangle \Theta(a) .
$$

Since the measure associated to $\Theta$ has no mass on proper analytic subsets of $X$, by Theorem 1.2, for $\Theta$-almost every $a$, we have $\mu_{n}^{a} \rightarrow \mu$. So the last sum converges to

$$
\int_{a \in X}\langle\mu, u(\cdot, a)\rangle \Theta(a)=\left\langle\pi_{1}^{*}(\mu), \Phi\right\rangle .
$$

The lemma holds in this case.

Case 2. Assume that $\Omega$ is of bidegree $(l, l)$ with $l \geq 1$ and $p=0$. For simplicity, we can assume that $\alpha=1, \beta=1,|u| \leq 1, \Omega\left(z_{1}\right) \leq \omega^{l}\left(z_{1}\right)$ and $\Theta\left(z_{2}\right) \leq \omega^{k-l}\left(z_{2}\right)$. Since $\left\langle\pi_{1}^{*}(\mu), \Phi\right\rangle=0$ because of bidegree reason on variable $z_{1}$, we have to verify that $\left\langle d_{t}^{-n}\left[\Gamma_{n}\right], \Phi\right\rangle \rightarrow 0$. We have

$$
\left|\left\langle d_{t}^{-n}\left[\Gamma_{n}\right], \Phi\right\rangle\right| \leq\left\langle d_{t}^{-n}\left[\Gamma_{n}\right], \omega\left(z_{1}\right)^{l} \wedge \omega\left(z_{2}\right)^{k-l}\right\rangle=d_{t}^{-n} \int_{X}\left(f^{n}\right)^{*}\left(\omega^{k-l}\right) \wedge \omega^{l} .
$$

Clearly, the last integral tends to 0 since $f$ is with dominant topological degree.

Case 3. In this last case, assume that $p \geq 1$. We also have $\left\langle\pi_{1}^{*}(\mu), \Phi\right\rangle=0$ because of bidegree reason on variable $z_{1}$. We check that $\left\langle d_{t}^{-n}\left[\Gamma_{n}\right], \Phi\right\rangle \rightarrow 0$. By Cauchy-Swcharz's inequality, we have

$$
\left|\left\langle d_{t}^{-n}\left[\Gamma_{n}\right], \Phi\right\rangle\right|^{2} \leq\left|\left\langle d_{t}^{-n}\left[\Gamma_{n}\right], \Phi_{1}\right\rangle\right|\left|\left\langle d_{t}^{-n}\left[\Gamma_{n}\right], \Phi_{2}\right\rangle\right|
$$

with

$\Phi_{1}:=|u|^{2} \alpha\left(z_{1}\right) \wedge \overline{\alpha\left(z_{1}\right)} \wedge \Omega\left(z_{1}\right) \wedge \Theta\left(z_{2}\right) \quad$ and $\quad \Phi_{2}:=\beta\left(z_{2}\right) \wedge \overline{\beta\left(z_{2}\right)} \wedge \Omega\left(z_{1}\right) \wedge \Theta\left(z_{2}\right)$.

Using the previous cases, we see that the first factor in the last product tend to 0 and the second one is bounded. The lemma follows. 
End of the proof of Proposition 5.1. By Proposition 2.1 and Lemma 5.2, it is enough to check that $\left\{\pi_{1}^{*}(\mu)\right\} \smile\{\Delta\}=1$ and that the h-tangent dimension of $\pi_{1}^{*}(\mu)$ with respect to $\Delta$ is 0 . Since $\mu$ is a probability measure, its class in $H^{k, k}(X)$ is also the class of a point. So the class of $\pi_{1}^{*}(\mu)$ is also the class of fiber of $\pi_{1}$. Any fiber of $\pi_{1}$ intersects $\Delta$ transversally at a point. So $\left\{\pi_{1}^{*}(\mu)\right\} \smile\{\Delta\}=1$.

To calculate the h-tangent dimension of $\pi_{1}^{*}(\mu)$, consider a point $a$ in $X$. We identify a neighbourhood of $a$ with a domain $U$ in $\mathbb{C}^{k}$ endowed with the standard coordinates $z=\left(z_{1}, \ldots, z_{k}\right)$. They induce a local coordinate system $\left(z, z^{\prime}\right)$ on a neighbourhood of the point $(a, a)$ in $\Delta$. We use a new coordinate system $\left(z, z^{\prime \prime}\right)$ with $z^{\prime \prime}:=z^{\prime}-z$. In these coordinates, a neighbourhood of $(a, a)$ is identified to an open subset of $U \times \mathbb{C}^{k}, \Delta$ is given by $\left\{z^{\prime \prime}=0\right\}$ and $\pi_{1}$ is always the natural projection onto $U$. The normal vector bundle of $\Delta$ is identified to $U \times \mathbb{C}^{k}$. The identity map is an admissible local map. So it is not difficult to see that the tangent current of $\pi_{1}^{*}(\mu)$ along $\Delta$ is also identified to $\pi_{1}^{*}(\mu)$. The h-dimension of this current is clearly 0 . This completes the proof of the proposition.

End of the proof of Theorem 1.1. We can now follow the proof for the case of holomorphic maps presented in [14]. For the reader's convenience, we give here the details.

First observe that with the notation as in Theorem 1.1, Proposition 5.1 implies that any cluster value of the sequence $\mu_{n}:=d_{t}^{-n} \sum_{a \in Q_{n}} \delta_{a}$ is a positive measure of mass at most equal to 1 . Therefore, it suffices to consider the case where $Q_{n}$ is the smallest set, i.e. the intersection of $R P_{n}$ with the support $\operatorname{supp}(\mu)$ of $\mu$. Fix a small constant $\nu>0$. It suffices to prove that any cluster value $\mu^{\prime}$ of $\mu_{n}$ satisfies $\mu^{\prime} \geq(1-4 \nu) \mu$. Let $B$ be an open subset of $X$. We have to prove that $\mu^{\prime}(B) \geq(1-4 \nu) \mu(B)$.

Recall that $\mu$ has no mass on proper analytic subsets of $X$. So it has no mass on $I_{\infty} \cup I_{\infty}^{\prime}$ nor on $\{\nu(R, \cdot)>0\}$. In what follows, we only consider balls whose centers stay outside these sets and belong to $\operatorname{supp}(\mu)$, in particular, we have $\nu(R, a)=0$ for such a center $a$. By Proposition 3.1, any small enough ball centered at $a$ admits at least $(1-\nu) d_{t}^{n}$ inverse branches of order $n$ of diameter $\leq\left(d_{k-1} / d_{t}+\epsilon\right)^{n / 2}$. The constant $\epsilon$ is fixed so that $d_{k-1} / d_{t}+\epsilon<1$. We only consider such balls.

Since these balls cover a set of full $\mu$ measure, we can find in $B$ a disjoint union of open sets of total $\mu$ measure such that each of these open sets is contained in one of the above considered balls and is biholomorphic to a cube in $\mathbb{C}^{k}$. Therefore, for simplicity, we can assume that $B$ is such a cube. We only have to check for $\mu$-almost every point $a \in X$ that $\# Q_{n} \cap B \geq(1-4 \nu) d_{t}^{n} \mu(B)$ when $n$ is large enough.

Choose a finite family of balls $B_{i}$ of center $b_{i}$ with $1 \leq i \leq m$ such that $\mu\left(B_{1} \cup \ldots \cup B_{m}\right)>1-\nu$ and each $B_{i}$ admits $(1-\nu \mu(B)) d_{t}^{n}$ inverse branches of order $n$ with diameter $\leq\left(d_{k-1} / d_{t}+\epsilon\right)^{n / 2}$ for $n$ large enough. Choose balls $B_{i}^{\prime} \Subset B_{i}$ such that $\mu\left(B_{1}^{\prime} \cup \ldots \cup B_{m}^{\prime}\right)>1-\nu$. 
Fix a constant $N$ large enough. Since $d_{t}^{-n}\left(f^{n}\right)^{*}\left(\delta_{a}\right)$ converge to $\mu$ for a generic point $a$ in $B$, the fiber $f^{-N}(a)$ contains at least $(1-\nu) d_{t}^{N}$ points in $\cup B_{i}^{\prime}$. Therefore, $B$ admits at least $(1-2 \nu) d_{t}^{N}$ inverse branches of order $N$ with small diameters whose images intersect $\cup B_{j}^{\prime}$. So the image of such a branch should be contained in one of the $B_{j}$. Choose an open set $B^{\prime} \subset B$ such that $\mu\left(B^{\prime}\right)>(1-\nu) \mu(B)$. In the same way, we show that for $n$ large enough, each $B_{j}$ admits $(1-2 \nu) \mu(B) d_{t}^{n-N}$ inverse branches of order $n-N$ whose images intersect $B^{\prime}$ and hence are contained in $B$. Observe that composing an inverse branch of order $N$ of $B$ whose image is contained in $B_{j}$ with an inverse branch of order $n-N$ of $B_{j}$ whose image is contained in $B$, we obtain an inverse branch of order $n$ of $B$ whose image is contained in $B$. Consequently, it follows that $B$ admits at least $(1-2 \nu)^{2} \mu(B) d_{t}^{n}$ inverse branches $g_{i}: B \rightarrow B^{(i)}$ of order $n$ with image $B^{(i)} \Subset B$.

Every holomorphic map $g: U \rightarrow V \Subset U$ on a convex open subset $U$ of $\mathbb{C}^{k}$ contracts the Kobayashi metric on $U$ and then admits an attractive fixed point $z$. Moreover, $g^{l}$ converges uniformly to $z$ and $\cap_{l \geq 0} g^{l}(U)=\{z\}$. Therefore, each $g_{i}$ admits a fixed attractive point $a^{(i)}$. This point is fixed and repelling for $f^{n}$. They are different since the $B^{(i)}$ are disjoint. Moreover, by definition of inverse branches, the orbit of $a^{(i)}$ does not intersect $\Sigma_{0} \supset I$. So this is a repelling periodic point of period $n$ for $f$ in our sense.

Finally, since $\mu$ is totally invariant, its support satisfies $f^{-1}\left(\operatorname{supp}(\mu) \backslash I^{\prime}\right) \subset$ $\operatorname{supp}(\mu)$. Hence, $a^{(i)}$, which is equal to $\cap_{l \geq 0} g_{i}^{l}(\operatorname{supp}(\mu) \cap B)$, is necessarily in $\operatorname{supp}(\mu)$. We deduce that

$$
\# Q_{n} \cap B \geq(1-2 \nu)^{2} \mu(B) d_{t}^{n} \geq(1-4 \nu) d_{t}^{n} \mu(B) .
$$

This completes the proof.

Remark 5.3. By Schwarz's lemma, since the diameter of $B^{(i)}$ is smaller than or equal to $\left(d_{k-1} / d_{t}+\epsilon\right)^{n / 2}$ all eigenvalues of the differential of $g_{i}$ at $a^{(i)}$ have modulus smaller than or equal to this constant. We deduce that the eigenvalues of the differential of $f^{n}$ at $a^{(i)}$ have modulus larger than or equal to $\left(d_{k-1} / d_{t}+\epsilon\right)^{-n / 2}$. Denote by $Q_{n}^{\epsilon}$ the set of repelling periodic points in $Q_{n}$ satisfying the last property. We then have

$$
d_{t}^{-n} \sum_{a \in Q_{n}^{\epsilon}} \delta_{a} \rightarrow \mu .
$$

Using Proposition 3.1, we obtain the following result as in the case of holomorphic maps.

Theorem 5.4. Let $f: X \rightarrow X, d_{t}, d_{p}$ and $\mu$ be as in Introduction. Then the measure $\mu$ is hyperbolic. Its Lyapounov exponents are bounded from below by $\frac{1}{2} \log \frac{d_{t}}{d_{k-1}}$ which is a strictly positive number.

The result was stated for projective manifolds in [19] but its proof is incomplete since the author uses again his lemma mentioned in the introduction. It was 
also stated in [14] for the general case of compact Kähler manifolds. We give here the details for the reader's convenience, see [14, Th. 1.120]. Note that the result can be also deduced from a more recent theorem by de Thélin [8]. The cases of endomorphisms of $\mathbb{P}^{k}$ and of polynomial-like maps were obtained in [2, 10]. See also [23] for the dimension 1 case.

Proof. Recall that quasi-p.s.h. functions are $\mu$-integrable. Let $J(f)$ be the Jacobian of $f$ with respect to the Kähler metric $\omega$ on $X$. Then, using a resolution of singularity for the graph of $f$ and local holomorphic coordinates, it is not difficult to show that $|\log J(f)| \leq|\varphi|$ for some quasi-p.s.h. function $\varphi$. So $|\log J(f)|$ is integrable with respect to $\mu$ and therefore, we can apply Oseledec's theorem to the natural extension of $f$ (a natural invertible map defined on the space of backward orbits of $f$ ) [21]. We deduce from this result that the smallest Lyapounov exponent of $\mu$ is equal to

$$
\chi:=\lim _{n \rightarrow \infty}-\frac{1}{n} \log \left\|D f^{n}(x)^{-1}\right\|
$$

for $\mu$-almost every $x$, where $D f^{n}$ denotes the differential of $f^{n}$.

Fix a small constant $\epsilon>0$. By Proposition 3.1, there is a ball $B$ of positive $\mu$ measure which admits at least $\frac{1}{2} d_{t}^{n}$ inverse branches $g_{i}: B \rightarrow B_{-n}^{(i)}$ of order $n$ with diameter $\leq\left(\frac{d_{k-1}}{d_{t}}+\epsilon\right)^{n / 2}$. By Cauchy's formula, if we reduce slightly the ball $B$, we can assume that $\left\|D g_{i}\right\| \leq A\left(\frac{d_{k-1}}{d_{t}}+\epsilon\right)^{n / 2}$ for some constant $A>0$. We then deduce that $\left\|\left(D f^{n}\right)^{-1}\right\| \leq A\left(\frac{d_{k-1}}{d_{t}}+\epsilon\right)^{n / 2}$ on $B_{-n}^{(i)}$.

The union $V_{n}$ of the $B_{-n}^{(i)}$ is of measure at least equal to $\frac{1}{2} \mu(B)$ since $\mu$ is totally invariant. Therefore, by Fatou's lemma,

$$
\frac{1}{2} \mu(B) \leq \limsup _{n \rightarrow \infty}\left\langle\mu, \mathbf{1}_{V_{n}}\right\rangle \leq\left\langle\mu, \limsup \mathbf{1}_{V_{n}}\right\rangle=\left\langle\mu, \mathbf{1}_{\limsup V_{n}}\right\rangle .
$$

Hence, for $x$ in the set $K:=\limsup V_{n}$, which has positive $\mu$ measure, we have $\left\|\left(D f^{n}\right)^{-1}\right\| \leq A\left(\frac{d_{k-1}}{d_{t}}+\epsilon\right)^{n / 2}$ for infinitely many of $n$. Hence, $\chi \geq \frac{1}{2} \log \left(\frac{d_{t}}{d_{k-1}+\epsilon d_{t}}\right)$. We obtain the result by letting $\epsilon$ to 0 .

\section{References}

[1] Blanchard A., Sur les variétés analytiques complexes. Ann. Sci. Éc. Norm. Sup. (3) 73 (1956), 157-202.

[2] Briend J.-Y., Duval J., Exposants de Liapunoff et distribution des points périodiques d'un endomorphisme de $\mathbb{C P}^{k}$. Acta Math. 182 (1999) (2), 143-157.

[3] — Deux caractérisations de la mesure d'équilibre d'un endomorphisme de $\mathbb{P}^{k}(\mathbb{C})$. Publ. Math. Inst. Hautes Études Sci. No. 93 (2001), 145-159. 
[4] Brolin H., Invariant sets under iteration of rational functions. Ark. Mat. 6 (1965), 103-144.

[5] Buff X., La mesure d'équilibre d'un endomorphisme de $\mathbb{P}^{k}(\mathbb{C})$ (d'après Briend et Duval). Séminaire Bourbaki. Vol. 2004/2005. Astérisque No. 307 (2006), Exp. No. 939, vii, 33-69.

[6] Demailly J.-P., Pseudoconvex-concave duality and regularization of currents, in: Several Complex Variables (Berkeley, CA, 1995-1996), in: Math. Sci. Res. Inst. Publ., vol. 37, Cambridge Univ. Press, Cambridge, 1999, 233-271.

[7] —_, Complex analytic and differential geometry, available at http://www.fourier.ujf-grenoble.fr/ demailly.

[8] De Thélin H., Sur les exposants de Lyapounov des applications méromorphes. Invent. math. 172 (2008), no. 1, 89-116.

[9] Dinh T.-C., Nguyen V.-A., The mixed Hodge-Riemann bilinear relations for compact Kähler manifolds. Geom. Funct. Anal., Vol. 16 (2006), 838-849.

[10] Dinh T.-C., Sibony N., Dynamique des applications d'allure polynomiale. J. Math. Pures Appl. (9) 82 (2003), no. 4, 367-423.

[11] — - Une borne supérieure pour l'entropie topologique d'une application rationnelle. Ann. of Math. (2) 161 (2005), 1637-1644.

[12] — Regularization of currents and entropy. Ann. Sci. École Norm. Sup. (4) 37 (2004), no. 6, 959-971.

[13] — - Distribution des valeurs d'une suite de transformations méromorphes et applications. Comment. Math. Helv. 81 (2006), 221-258.

[14] —-, Dynamics in several complex variables: endomorphisms of projective spaces and polynomial-like mappings. Holomorphic dynamical systems, 165-294, Lecture Notes in Math., 1998, Springer, Berlin, 2010.

[15] — Density of positive closed currents and dynamics of Hénon-type automorphisms of $\mathbb{C}^{k}$ (part I). arXiv:1203.5810, 50 pages.

[16] Fornæss, J.-E., Sibony N., Complex dynamics in higher dimensions. Notes partially written by Estela A. Gavosto. NATO Adv. Sci. Inst. Ser. C Math. Phys. Sci., 439, Complex potential theory (Montreal, PQ, 1993), 131-186, Kluwer Acad. Publ., Dordrecht, 1994.

[17] Freire A., Lopes A., Mañé R., An invariant measure for rational maps. Bol. Soc. Brasil. Mat. 14 (1983), no. 1, 45-62.

[18] Gromov M., Convex sets and Kähler manifolds. Advances in differential geometry and topology, Word Sci. Publishing, Teaneck, NJ, 1998, 1-38. 
[19] Guedj V., Ergodic properties of rational mappings with large topological degree. Ann. of Math. (2) 161 (2005), no. 3, 1589-1607.

[20] Hörmander L., The analysis of linear partial differential operators. I. Distribution theory and Fourier analysis. Grundlehren der Mathematischen Wissenschaften, 256. Springer-Verlag, Berlin, 1983.

[21] Katok A., Hasselblatt B., Introduction to the modern theory of dynamical systems. Encyclopedia of Mathematics and its Applications, 54. Cambridge University Press, Cambridge, 1995.

[22] Khovanskii A.G., The geometry of convex polyhedra and algebraic geometry. Uspehi Mat. Nauk. 34:4 (1979), 160-161.

[23] Lyubich M. Ju., Entropy properties of rational endomorphisms of the Riemann sphere. Ergodic Theory Dynam. Systems 3 (1983), no. 3, 351-385.

[24] Sibony N., Dynamique des applications rationnelles de $\mathbb{P}^{k}$. In Dynamique et géométrie complexes (Lyon, 1997), 97-185, Panoramas et Synthèses 8, Soc. Math. France, Paris, (1999).

[25] Sibony N., Wong P., Some results on global analytic sets. Séminaire Pierre LelongHenri Skoda (Analyse), année 1978/1979, 221-237, Lecture Notes in Math. 822, Springer, Berlin (1980).

[26] Siu Y.T., Analyticity of sets associated to Lelong numbers and the extension of closed positive currents. Invent. Math. 27 (1974), 53-156.

[27] Teissier B., Du théorème de l'index de Hodge aux inégalités isopérimétriques. $C$. R. Acad. Sci. Paris Sér. A-B 288 (1979), no. 4, 287-289.

[28] Timorin V.A., Mixed Hodge-Riemann bilinear relations in a linear context. Funct. Anal. Appl. 32:4 (1998), 268-272.

[29] Tortrat P., Aspects potentialistes de l'itération des polynômes. Séminaire de Théorie du Potentiel, Paris, No. 8, 195-209, Lecture Notes in Math., 1235, Springer, Berlin, 1987.

[30] Voisin C., Hodge theory and complex algebraic geometry. I. Cambridge Studies in Advanced Mathematics, 76, Cambridge University Press, Cambridge, 2002.

T.-C. Dinh, UPMC Univ Paris 06, UMR 7586, Institut de Mathématiques de Jussieu, 4 place Jussieu, F-75005 Paris, France.

DMA, UMR 8553, Ecole Normale Supérieure, 45 rue d'Ulm, 75005 Paris, France.

dinh@math.jussieu.fr, http://www.math.jussieu.fr/ dinh

V.-A. Nguyên, Mathématique-Bâtiment 425, UMR 8628, Université Paris-Sud, 91405 Orsay, France.

VietAnh.Nguyen@math.u-psud.fr, http://www.math.u-psud.fr/ vietanh

T.-T. Truong, Department of Mathematics, Syracuse University, Syracuse, NY 13244, USA tutruong@syr.edu 